Case Report

\title{
Varicella-Zoster Gastritis in a Liver Transplant Patient: A Call to Attention
}

Víctor Escrich ${ }^{1,}{ }^{*}$, Ángela Martinez ${ }^{1}$, Berta Lapeña ${ }^{1}$, Marta Mayorga ${ }^{2}$, Antonio Cuadrado ${ }^{3}$, Javier Crespo $^{3}$, Emilio Fábrega ${ }^{3}$

1. Gastroenterology and Hepatology Unit, Hospital San Pedro, C/Piqueras 98, Logroño 26006, La Rioja, Spain; E-Mails: vescrich@riojasalud.es; amartinezh@riojasalud.es; blapena@riojasalud.es

2. Department of Pathology, Hospital Universitario Marqués de Valdecilla, Avenida Valdecilla s/n, Santander 39008, Cantabria, Spain; E-Mail: marta.mayorga@scsalud.es

3. Gastroenterology and Hepatology Unit, Hospital Universitario Marqués de Valdecilla, Instituto de Investigación Marqués de Valdecilla (IDIVAL), Avenida Valdecilla s/n, Santander 39008, Cantabria, Spain; E-Mails: antonio.cuadrado@scsalud.es; javier.crespo@scsalud.es; emilio.fabrega@scsalud.es

* Correspondence: Víctor Escrich; E-Mail: vescrich@riojasalud.es

Academic Editor: Tony C. K. Tham

OBM Hepatology and Gastroenterology

2021, volume 5, issue 2

doi:10.21926/obm.hg.2102057
Received: March 01, 2021

Accepted: June 10, 2021

Published: June 21, 2021

\begin{abstract}
The reactivation of the Varicella-zoster virus (VZV) is a rare cause of acute gastritis in adults. About 30 cases have been reported in the literature, mostly with immunocompromised patients and mainly after bone marrow transplantation or during the development of malignant hematological diseases. Clinically, it is usually accompanied by cutaneous manifestations. Here, we studied a case of VZV gastritis in a liver transplant (LT) patient. We described the main symptoms, endoscopic findings, histologic changes, and treatment of VZV gastritis. Till now, no case of acute gastritis due to the reactivation of VZV after solid organ transplantation had been reported [2-5]. This was the first reported case of acute gastritis by the reactivation of VZV after LT without cutaneous vesicular eruption. Gastrointestinal symptoms usually develop a week before the onset of fever and cutaneous manifestations. However, in some cases, like this one, vesicular rashes may be absent,
\end{abstract}

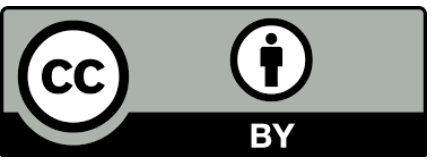

(C) 2021 by the author. This is an open access article distributed under the conditions of the Creative Commons by Attribution License, which permits unrestricted use, distribution, and reproduction in any medium or format, provided the original work is correctly cited. 
making the diagnosis quite challenging. In conclusion, through this case, we suggest including VZV gastritis in the differential diagnosis of gastrointestinal symptoms after transplantation and informing about the response of VZV gastritis to treatment with oral acyclovir.

\section{Keywords}

Varicella-zoster gastritis; liver transplant

The reactivation of the Varicella-zoster virus (VZV) is a rare cause of acute gastritis in adults. About 30 cases have been reported in the literature, mostly with immunocompromised patients and mainly after bone marrow transplantation or during the development of malignant hematological diseases. Clinically, it is usually accompanied by cutaneous manifestations [1-3].

In this study, we reported the case of a 43-year-old man, who had severe epigastric pain, nausea, early satiety, postprandial fullness, anorexia, and weight loss of over $10 \mathrm{~kg}$ in three weeks. Those symptoms remained unresolved after the administration of omeprazole. The subject had undergone liver transplantation (LT) for hepatitis $C$ virus five years ago. The hepatitis $C$ disease was treated in the second year after LT with peginterferon alfa- $2 \mathrm{~b}$ and ribavirin for 12 months, and a sustained virologic response was achieved. In the past three years, the patient had not experienced any episode of rejection and did not need an increase in immunosuppression. The patient had been receiving tacrolimus monotherapy at a stable level of $5 \mu \mathrm{g} / \mathrm{L}$ for the past year. Thus, the patient was not over-immunosuppressed and did not have any comorbidities such as zoster reactivation, diabetes mellitus, or HIV infection.

The patient's vital signs were stable and abdominal, chest, and skin physical examination results were normal. Results of laboratory tests and computed tomography of the abdomen were also normal. Upper gastrointestinal endoscopy (UGE) revealed erythematous mucosa with multiple raised lesions, which showed nodular appearance between 3 and $10 \mathrm{~mm}$ in size, umbilicated at the center, and covered by fibrin (Figure 1a). The rapid urease test for Helicobacter pylori showed negative results. Upon suspecting that it might be a case of cytomegalovirus (CMV) gastritis, we started treatment with oral valganciclovir (900 mg/12 h). Histopathology of the biopsy specimen suggested non-specific acute gastritis. No viral inclusions were observed (Figure 2). Other microorganisms were excluded, and immunohistochemistry for CMV and VZV showed negative results. Tests for the presence of IgM antibodies for VZV and CMV also showed negative results. Furthermore, polymerase chain reaction (PCR) and culture for CMV showed negative results, and hence, valganciclovir was discontinued after two weeks of administration. 


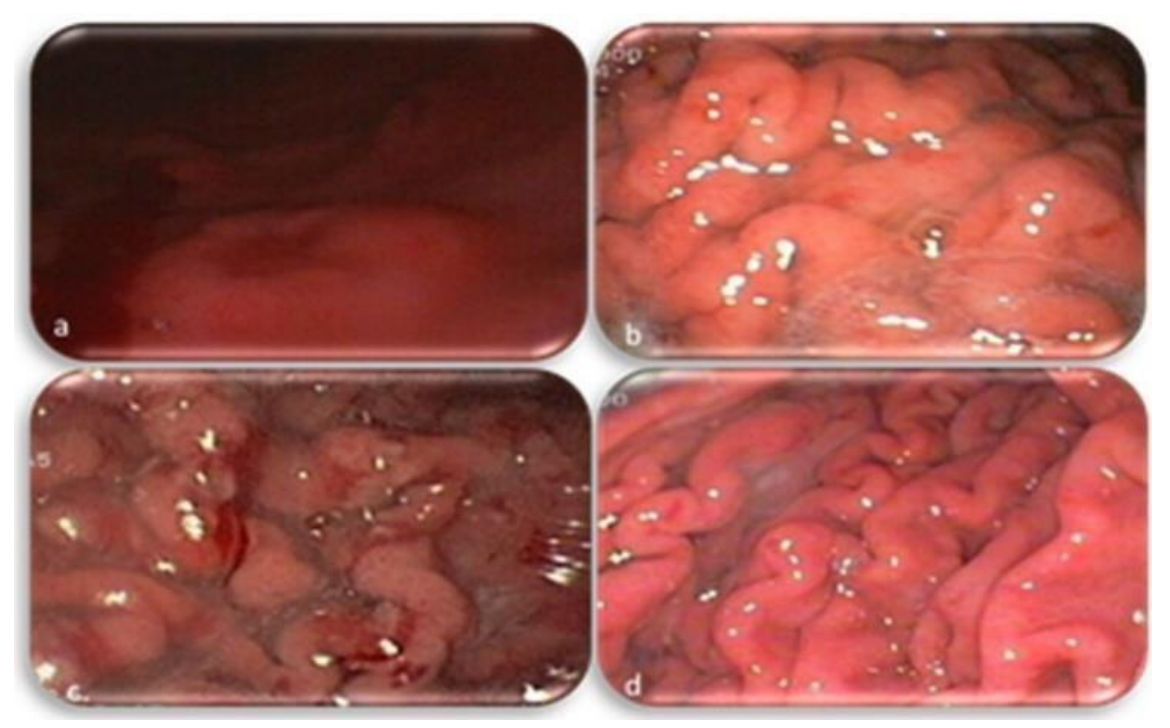

Figure 1 Endoscopic findings showing: a. Gastric antrum: Erythematous mucosa with raised lesions, with nodular appearance between 3 and $10 \mathrm{~mm}$ in size, umbilicated at the center. b. Gastric body: Thickened gastric folds and patchy erythema. c. Gastric body after valganciclovir treatment: Worsening of gastritis with spontaneous mucosal bleeding. d. Gastric body: Improvement of gastritis one month after acyclovir treatment.

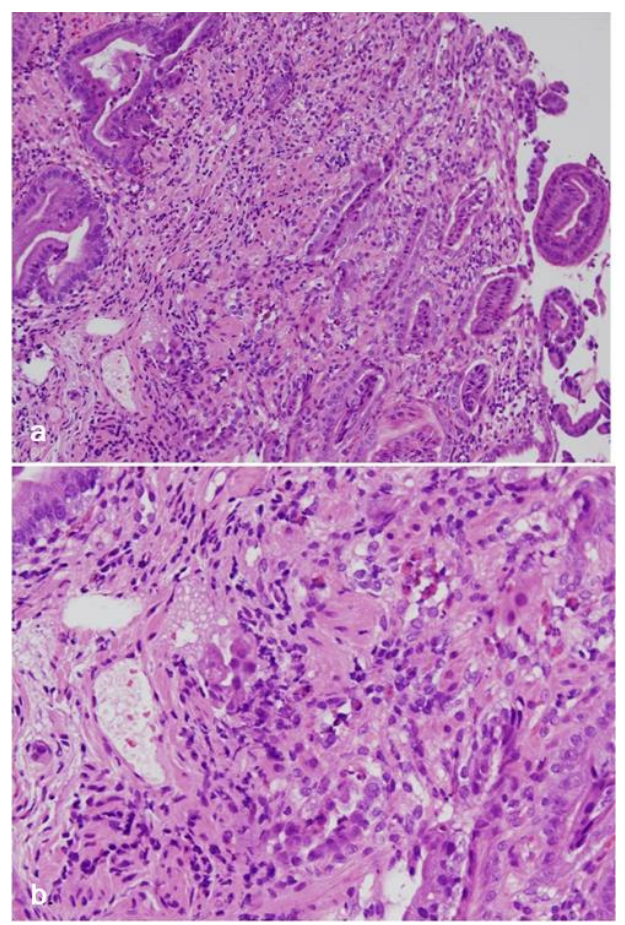

Figure 2 a. Histological sections stained with hematoxylin and eosin, showing acute inflammation with the presence of abundant eosinophils and signs of epithelial regeneration and foveolar hyperplasia; magnification at 10x. No viral inclusions were observed, and immunohistochemistry for cytomegalovirus and Varicella-zoster virus showed negative results. No other microorganisms were identified. b. Magnification at $20 x$. 
Due to the persistence of the infection, another UGE was performed (Figure 1c). Gastric biopsy material tested positive for VZV DNA using the nested PCR method. Oral acyclovir ( $800 \mathrm{mg} / 6 \mathrm{~h}$ ) was administered for 14 days without modification of the immunosuppressive therapy. On the $10^{\text {th }}$ day, the patient showed complete remission of the aforementioned symptoms (including gastroparesis). A follow-up UGE performed one month later showed normalization of the gastric mucosa.

Till now, no cases of acute gastritis reactivation of VZV after solid organ transplantation have been reported [2-5]. This was the first reported case of acute gastritis reactivation of VZV after LT without cutaneous vesicular eruption. It is important to note that only the stomach was infected, without any other systemic manifestation. Gastrointestinal symptoms usually develop a week before the onset of fever and cutaneous manifestations; however, in some cases, like this one, vesicular rashes might be absent, making the diagnosis quite challenging [2]. Symptoms related to intestinal motility have been described [2]. In this study, the occurrence of postprandial fullness or gastroparesis could have been related to the effect of the VZV on the secondary neuronal plexus and mesenteric sympathetic ganglia [2].

Endoscopic findings in the literature had reported the presence of enlarged gastric folds, erosions in the stomach, ectatic blood vessels at the surface of the mucosa, and papules in the duodenum [4]. It is important to remember that histological findings may be non-specific and the absence of demonstrable intranuclear inclusions or VZV by immunohistochemistry does not exclude the diagnosis. Therefore, to detect the presence of low numbers of viral particles, additional methods such as PCR are required [3].

In conclusion, this case suggests including VZV gastritis in the differential diagnosis of gastrointestinal symptoms after transplant and demonstrates an excellent response to the treatment of VZV gastritis with oral acyclovir.

\section{Author Contributions}

Escrich V, Martinez A, Lapeña B, Mayorga M, Cuadrado A, Crespo J, and Fábrega E solely contributed to this letter.

\section{Competing Interests}

The authors have declared that no competing interests exist.

\section{References}

1. Atkinson K, Meyers JD, Storb R, Prentice RL, Thomas ED. Varicella-zoster virus infection after marrow transplantation for aplastic anaemia or leukaemia. Transplantation. 1980; 29: 47-50.

2. Serris A, Michot JM, Fourn E, Bras PL, Dollat M, Hirsch G, et al. Infection disséminée au virus varicelle-zona avec gastrite hémorragique compliquant une leucémie lymphoïde chronique: Uneobservation et revue de la litterature. Rev Med Interne. 2014; 35: 337-340.

3. McCluggage WG, Fox JD, Baillie KE, Coyle PV, Jones FG, O'Hara MD. Varicella zoster gastritis in a bone marrow transplant recipient. J Clin Pathol. 1994; 47: 1054-1056.

4. Ugras $M$, Vitrinel A, Yilmaz G, Midilli K, Ozkan F. Varicella gastritis in an inmunocompetent child. J Clin Virol. 2013; 56: 153-155. 
5. Nohr EW, Itani DM, Andrews CN, Kelly MM. Varicella-zoster virus gastritis: Case report and review of the literature. Int J Surg Pathol. 2017; 25: 449-452.

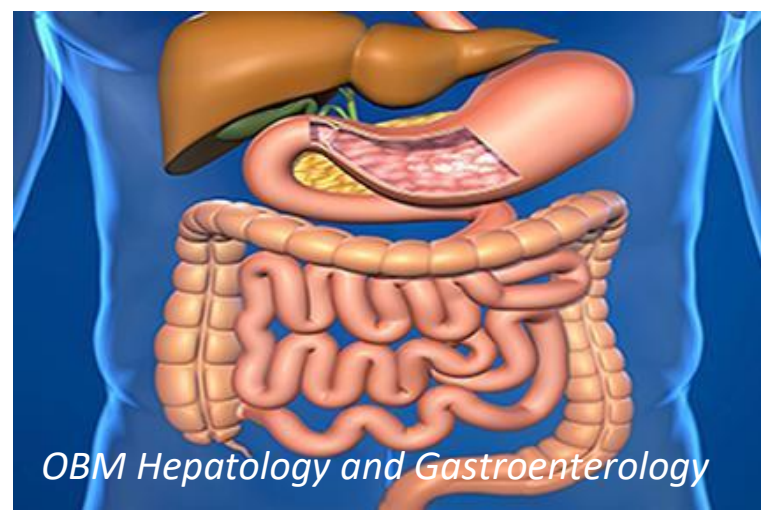

Enjoy OBM Hepatology and Gastroenterology by:

1. Submitting a manuscript

2. Joining in volunteer reviewer bank

3. Joining Editorial Board

4. Guest editing a special issue

For more details, please visit:

http://www.lidsen.com/journals/hg 